\title{
Inverse boundary value problem for Schrödinger equation in two dimensions
}

\author{
M. Yamamoto*and O. Yu. Imanuvilov ${ }^{\dagger}$
}

\begin{abstract}
We relax the regularity condition on potentials of the Schrödinger equation in uniqueness results on the inverse boundary value problem which were recently proved in [11] and [5].
\end{abstract}

Let $\Omega \subset \mathbb{R}^{2}$ be a bounded smooth domain with $\partial \Omega=\cup_{j=0}^{K} \Sigma_{j}$ where $\Sigma_{j}$ are smooth contours and $\Sigma_{0}$ is the external contour. Let $\nu=\left(\nu_{1}, \nu_{2}\right)$ be the unit outer normal to $\partial \Omega$ and let $\frac{\partial}{\partial \nu}=\nabla \cdot \nu$.

In this domain we consider the Schrödinger equation with some potential $q$ :

$$
(\Delta+q) u=0 \text { in } \Omega .
$$

Let $\widetilde{\Gamma}$ be a non-empty arbitrary fixed relatively open subset of $\partial \Omega$. Denote $\Gamma_{0}=\operatorname{Int}(\partial \Omega \backslash \widetilde{\Gamma})$. Consider the partial Cauchy data

$$
\mathcal{C}_{q}=\left\{\left.\left(u, \frac{\partial u}{\partial \nu}\right)\right|_{\widetilde{\Gamma}} ;(\Delta+q) u=0 \quad \text { in } \Omega,\left.u\right|_{\Gamma_{0}}=0,\left.u\right|_{\widetilde{\Gamma}}=f\right\} .
$$

The goal of this article is to improve the regularity assumption on the potential $q$ in the case of arbitrary subboundary $\widetilde{\Gamma}$ for the uniqueness result in the inverse problem of recovery of potential from the partial data (2). In the case of $\widetilde{\Gamma}=\partial \Omega$, this inverse problem was formulated by Calderón in [7. Under the assumption $q \in C^{4+\alpha}(\bar{\Omega})$ the result was proved in Imanuvilov, Uhlmann and Yamamoto [11. In Guillarmou and Tzou [10], the assumption on potentials was improved up to $C^{2+\alpha}(\bar{\Omega})$.

In particular, in the two-dimensional full Cauchy data case of $\widetilde{\Gamma}=\partial \Omega$, we refer to Astala and Päivärinta [1], Blasten [2, Brown and Uhlmann [4, Bukhgeim [5], Nachman [14]. In [2], the full Cauchy data uniquely determine the potential within $W_{p}^{1}(\Omega)$ with $p>2$. As for the related problem of recovery of the conductivity, [1] proved the uniqueness result for conductivities from $L^{\infty}(\Omega)$, improving the result of [14]. We also mention that for the case of full Cauchy data a relaxed regularity assumption on potential was claimed in [5] but the proof itself is missing some details.

In three or higher dimensions, for the full Cauchy data, Sylvester and Uhlmann [16] proved the uniqueness of recovery of conductivity in $C^{2}(\bar{\Omega})$, and later the regularity assumption was relaxed up to $C^{\frac{3}{2}}(\bar{\Omega})$ in Päivärinta, Panchenko and Uhlmann [15] and up to $W_{p}^{\frac{3}{2}}(\Omega)$ with $p>2 n$ in Brown and Torres [3]. For the case of partial Cauchy data, uniqueness theorems were proved under assumption that a potential of the Schrödinger equation belongs to $L^{\infty}(\Omega)$ (see Bukhgeim and Uhlmann [6], Kenig, Sjöstrand and Uhlmann [13]).

Our main result is as follows

Theorem 1 Let $q_{1}, q_{2} \in C^{\alpha}(\bar{\Omega})$ for some $\alpha \in(0,1)$ if $\widetilde{\Gamma}=\partial \Omega$ and $q_{1}, q_{2} \in W_{p}^{1}(\Omega)$ for some $p>2$ otherwise. If $\mathcal{C}_{q_{1}}=\mathcal{C}_{q_{2}}$ then $q_{1}=q_{2}$.

\footnotetext{
*Department of Mathematical Sciences, University of Tokyo, Komaba, Meguro, Tokyo 153, Japan e-mail: myama@ms.utokyo.ac.jp

${ }^{\dagger}$ Department of Mathematics, Colorado State University, 101 Weber Building, Fort Collins, CO 80523-1874, U.S.A. E-mail: oleg@math.colostate.edu. This work is partially supported by NSF Grant DMS 0808130.
} 
The rest part of the paper is devoted to the proof of the theorem. Throughout the article, we use the following notations.

Notations. $i=\sqrt{-1}, x_{1}, x_{2} \in \mathbb{R}^{1}, z=x_{1}+i x_{2}, \bar{z}$ denotes the complex conjugate of $z \in \mathbb{C}$. We identify $x=\left(x_{1}, x_{2}\right) \in \mathbb{R}^{2}$ with $z=x_{1}+i x_{2} \in \mathbb{C} . \partial_{z}=\frac{1}{2}\left(\partial_{x_{1}}-i \partial_{x_{2}}\right), \partial_{\bar{z}}=\frac{1}{2}\left(\partial_{x_{1}}+i \partial_{x_{2}}\right), D=\left(\frac{1}{i} \partial_{x_{1}}, \frac{1}{i} \partial_{x_{2}}\right)$. The tangential derivative on the boundary is given by $\partial_{\vec{\tau}}=\nu_{2} \frac{\partial}{\partial x_{1}}-\nu_{1} \frac{\partial}{\partial x_{2}}$, where $\nu=\left(\nu_{1}, \nu_{2}\right)$ is the unit outer normal to $\partial \Omega$.

\section{Proof.}

First Step.

Let $\Phi=\varphi+i \psi$ be a holomorphic function on $\Omega$ such that $\varphi, \psi$ are real-valued and

$$
\Phi \in C^{2}(\bar{\Omega}),\left.\quad \operatorname{Im} \Phi\right|_{\Gamma_{0}}=0 .
$$

Denote by $\mathcal{H}$ the set of the critical points of the function $\Phi$. Suppose that this set is not empty, each critical point is nondegenerate, $\mathcal{H} \cap \bar{\Gamma}_{0}=\emptyset$ and

$$
\operatorname{mes}(\mathcal{J})=0, \quad \mathcal{J}=\left\{x ; \partial_{\vec{\tau}} \psi(x)=0, x \in \widetilde{\Gamma}\right\} .
$$

Here $\vec{\tau}$ is an unit tangential vector to $\partial \Omega$. Consider the operator $L_{q}(x, D)=-\sum_{j=1}^{2}\left(D_{j}+\tau i \varphi_{x_{j}}\right)^{2}+q$. It is known (see [12] Proposition 2.5) that there exists a constant $\tau_{0}$ such that for $|\tau| \geq \tau_{0}$ and any $f \in L^{2}(\Omega)$, there exists a solution to the boundary value problem

$$
L_{q}(x, D) u=f \quad \text { in } \Omega,\left.\quad u\right|_{\Gamma_{0}}=0
$$

such that

$$
\|u\|_{H^{1, \tau}(\Omega)} / \sqrt{|\tau|} \leq C\|f\|_{L^{2}(\Omega)}
$$

Moreover if $f / \partial_{z} \Phi \in L^{2}(\Omega)$, then for any $|\tau| \geq \tau_{0}$ there exists a solution to the boundary value problem (5) such that

$$
\|u\|_{H^{1, \tau}(\Omega)} \leq C\left\|f / \partial_{z} \Phi\right\|_{L^{2}(\Omega)} .
$$

The constants $C$ in (6) and (77) are independent of $\tau$. Here and henceforth we set

$$
\|u\|_{H^{1, \tau}(\Omega)}=\left(\|u\|_{H^{1}(\Omega)}^{2}+|\tau|^{2}\|u\|_{L^{2}(\Omega)}^{2}\right)^{\frac{1}{2}} .
$$

\section{Second Step.}

Here we will construct complex geometrical optics solutions. Henceforth by $o_{L^{2}(\Omega)}\left(\frac{1}{\tau}\right)$, we mean a function $f(\epsilon, \tau, \cdot) \in L^{2}(\Omega)$ such that $\lim _{\tau \rightarrow \infty}|\tau|\|f(\epsilon, \tau, \cdot)\|_{L^{2}(\Omega)}=0$ for all small $\epsilon>0$, and by $o\left(\frac{1}{\tau}\right)$, we mean $a(\epsilon, \tau)$ such that $\lim _{\tau \rightarrow \infty}|\tau||a(\epsilon, \tau)|=0$ for all small $\epsilon>0$.

Let $\left\{q_{1, \epsilon}\right\}_{\epsilon \in(0,1)}$ be a sequence of smooth functions converging to $q_{1}$ in $W_{p}^{1}(\Omega)$ or $C^{\alpha}(\bar{\Omega})$ (depending on the assumption on the regularity of $\left.q_{1}\right)$ such that $q_{1, \epsilon}=q_{1}$ on $\mathcal{H}$. Let $p_{\epsilon}$ be the complex geometrical optics solution to the Schrödinger operator $\Delta+q_{1, \epsilon}$ which we constructed in [11. The function $p_{\epsilon}$ can be written in the form:

$$
\begin{array}{r}
p_{\epsilon}(x)=e^{\tau \Phi}\left(a+a_{0, \epsilon} / \tau\right)+e^{\tau \bar{\Phi}} \overline{\left(a+b_{1, \epsilon} / \tau\right)} \\
-\left(e^{\tau \Phi} \frac{\left(\partial_{\bar{z}}^{-1}\left(a q_{1, \epsilon}\right)-M_{1, \epsilon}\right)}{4 \tau \partial_{z} \Phi}+e^{\tau \bar{\Phi}} \frac{\left(\partial_{z}^{-1}\left(\bar{a} q_{1, \epsilon}\right)-M_{3, \epsilon}\right)}{4 \tau \overline{\partial_{z} \Phi}}\right)+e^{\tau \varphi} O_{L^{2}(\Omega)}\left(\frac{1}{\tau}\right) \quad \text { as } \tau \rightarrow+\infty,
\end{array}
$$

where $a \in C^{6}(\bar{\Omega})$ is some holomorphic function on $\Omega$ such that $\left.\operatorname{Re} a\right|_{\Gamma_{0}}=0$. The operators $\partial_{z}^{-1}$ and $\partial_{\bar{z}}^{-1}$ are given by

$$
\partial_{\bar{z}}^{-1} g=-\frac{1}{\pi} \int_{\Omega} \frac{g(\zeta, \bar{\zeta})}{\zeta-z} d \xi_{2} d \xi_{1}, \quad \partial_{z}^{-1} g=\overline{\partial_{\bar{z}}^{-1} \bar{g}}
$$

Moreover for some $\widetilde{x} \in \mathcal{H}$, we assume that $a(\widetilde{x}) \neq 0$ and $a(x)=0$ for $x \in \mathcal{H} \backslash\{\widetilde{x}\}$, and the polynomials $M_{1, \epsilon}(z)$ and $M_{3, \epsilon}(\bar{z})$ satisfy

$$
\partial_{z}^{j}\left(\partial_{\bar{z}}^{-1}\left(a q_{1, \epsilon}\right)-M_{1, \epsilon}\right)(x)=0, \quad \partial_{\bar{z}}^{j}\left(\partial_{z}^{-1}\left(\bar{a} q_{1, \epsilon}\right)-M_{3, \epsilon}\right)(x)=0, \quad x \in \mathcal{H},
$$


$a_{0, \epsilon}, a_{1, \epsilon} \in C^{6}(\bar{\Omega})$ are holomorphic functions such that

$$
\left.\left(a_{0, \epsilon}+\bar{a}_{1, \epsilon}\right)\right|_{\Gamma_{0}}=\frac{\left(\partial_{\bar{z}}^{-1}\left(a q_{1, \epsilon}\right)-M_{1, \epsilon}\right)}{4 \partial_{z} \Phi}+\frac{\left(\partial_{z}^{-1}\left(\bar{a} q_{1, \epsilon}\right)-M_{3, \epsilon}\right)}{4 \overline{\partial_{z} \Phi}} .
$$

We look for a solution $u_{1}$ in the form $u_{1}=p_{\epsilon}+m_{\epsilon}$. Consider the equation

$$
L_{q_{1}}(x, D) u_{1}=L_{q_{1, \epsilon}}(x, D)\left(p_{\epsilon}+m_{\epsilon}\right)+\left(q_{1}-q_{1, \epsilon}\right)\left(p_{\epsilon}+m_{\epsilon}\right)=L_{q_{1}}(x, D) m_{\epsilon}+\left(q_{1}-q_{1, \epsilon}\right) p_{\epsilon}=0 .
$$

By (7) there exists a solution to the boundary value problem

$$
L_{q_{1}}(x, D) m_{\epsilon}+\left(q_{1}-q_{1, \epsilon}\right) p_{\epsilon}=0 \quad \text { in } \quad \Omega,\left.\quad m_{\epsilon}\right|_{\Gamma_{0}}=0
$$

such that

$$
\left\|m_{\epsilon}\right\|_{H^{1, \tau}(\Omega)} \leq C(\epsilon) \quad \forall \tau>\tau_{0}(\epsilon)
$$

where $C(\epsilon)$ is independent of $\tau$ and

$$
C(\epsilon) \rightarrow 0 \quad \text { as } \quad \epsilon \rightarrow 0 .
$$

Since the Cauchy data (2) for potentials $q_{1}$ and $q_{2}$, are equal, there exists a solution $u_{2}$ to the Schrödinger equation with the potential $q_{2}$ such that $u_{1}=u_{2}$ on $\partial \Omega$ and $\frac{\partial u_{1}}{\partial \nu}=\frac{\partial u_{2}}{\partial \nu}$ on $\widetilde{\Gamma}$. Setting $u=u_{1}-u_{2}$, we obtain

$$
\left(\Delta+q_{2}\right) u=\left(q_{2}-q_{1}\right) u_{1} \quad \text { in } \Omega,\left.\quad u\right|_{\partial \Omega}=\left.\frac{\partial u}{\partial \nu}\right|_{\widetilde{\Gamma}}=0 .
$$

In a way similar to the construction of $u_{1}$, we construct the complex geometrical optics solution $v$ for the Schrödinger equation with the potential $q_{2}$. The construction of $v$ repeats the corresponding steps of the construction of $u_{1}$. The only difference is that instead of $q_{1, \epsilon}$ and $\tau$, we use $q_{2, \epsilon}$ and $-\tau$ respectively. We provide details of the construction of $v$ for the sake of completeness.

Let $\left\{q_{2, \epsilon}\right\}_{\epsilon \in(0,1)}$ be a sequence of smooth functions converging to sufficiently close to $q_{2}$ in $W_{p}^{1}(\Omega)$ or $C^{\alpha}(\bar{\Omega})$ such that $q_{2, \epsilon}=q_{2}$ on $\mathcal{H}$. Let $\widetilde{p}_{\epsilon}$ be the complex geometrical optics solution to the Schrödinger operator $\Delta+q_{2, \epsilon}$ constructed in [1]:

$$
\begin{array}{r}
\tilde{p}_{\epsilon}(x)=e^{-\tau \Phi}\left(a+b_{0, \epsilon} / \tau\right)+e^{-\tau \bar{\Phi}} \overline{\left(a+b_{1, \epsilon} / \tau\right)} \\
+\left(e^{-\tau \Phi} \frac{\left(\partial_{\bar{z}}^{-1}\left(a q_{2, \epsilon}\right)-M_{2, \epsilon}\right)}{4 \tau \partial_{z} \Phi}+e^{-\tau \bar{\Phi}} \frac{\left(\partial_{z}^{-1}\left(\bar{a} q_{2, \epsilon}\right)-M_{4, \epsilon}\right)}{4 \tau \overline{\partial_{z} \Phi}}\right)+e^{-\tau \varphi} O_{L^{2}(\Omega)}\left(\frac{1}{\tau}\right),
\end{array}
$$

where $M_{2, \epsilon}(z)$ and $M_{4, \epsilon}(\bar{z})$ satisfy

$$
\partial_{z}^{j}\left(\partial_{\bar{z}}^{-1}\left(a q_{1, \epsilon}\right)-M_{2, \epsilon}\right)(x)=0, \quad \partial_{\bar{z}}^{j}\left(\partial_{z}^{-1}\left(\bar{a} q_{1, \epsilon}\right)-M_{4, \epsilon}\right)(x)=0, \quad x \in \mathcal{H} .
$$

and $b_{0, \epsilon}, b_{1, \epsilon}$ are holomorphic functions such that

$$
\left.\left(b_{0, \epsilon}+\bar{b}_{1, \epsilon}\right)\right|_{\Gamma_{0}}=-\frac{\left(\partial_{\bar{z}}^{-1}\left(a q_{2, \epsilon}\right)-M_{2, \epsilon}\right)}{4 \partial_{z} \Phi}-\frac{\left(\partial_{z}^{-1}\left(\bar{a} q_{2, \epsilon}\right)-M_{4, \epsilon}\right)}{4 \overline{\partial_{z} \Phi}} .
$$

We look for a solution $v$ in the form $v=\widetilde{p}_{\epsilon}+\widetilde{m}_{\epsilon}$. Consider the operator

$$
L_{q_{2}}(x, D) v=L_{q_{2, \epsilon}}(x, D)\left(\widetilde{p}_{\epsilon}+\widetilde{m}_{\epsilon}\right)+\left(q_{2}-q_{2, \epsilon}\right)\left(\widetilde{p}_{\epsilon}+\widetilde{m}_{\epsilon}\right)=L_{q_{2}}(x, D) \widetilde{m}_{\epsilon}+\left(q_{2}-q_{2, \epsilon}\right) \widetilde{p}_{\epsilon}=0 .
$$

By (77) there exists a solution to the boundary value problem

$$
L_{q_{2}}(x, D) \widetilde{m}_{\epsilon}+\left(q_{2}-q_{2, \epsilon}\right) \widetilde{p}_{\epsilon}=0 \quad \text { in } \quad \Omega,\left.\quad \widetilde{m}_{\epsilon}\right|_{\Gamma_{0}}=0
$$

such that

$$
\left\|\widetilde{m}_{\epsilon}\right\|_{H^{1, \tau}(\Omega)} \leq C(\epsilon) \quad \forall \tau>\tau_{0}(\epsilon)
$$

where $C(\epsilon)$ is independent of $\tau$ and

$$
C(\epsilon) \rightarrow 0 \quad \text { as } \quad \epsilon \rightarrow 0 .
$$




\section{Third Step.}

We will prove $q_{1}(\widetilde{x})=q_{2}(\widetilde{x})$ where $a(\widetilde{x}) \neq 0$ and $a(x)=0$ for $x \in \mathcal{H} \backslash\{\widetilde{x}\}$ in the case where $q_{1}, q_{2} \in W_{p}^{1}(\Omega)$.

Denote $q=q_{1}-q_{2}$. Taking the scalar product of equation (10) and the function $v$, we have:

$$
\int_{\Omega} q u_{1} v d x=0
$$

By (9) and (12)

$$
0=\int_{\Omega} q u_{1} v d x=\int_{\Omega} q p_{\epsilon} \widetilde{p}_{\epsilon} d x+K(\epsilon, \tau)
$$

where

$$
\overline{\lim }_{\tau \rightarrow+\infty} \tau|K(\epsilon, \tau)| \leq C(\epsilon), \quad C(\epsilon) \rightarrow 0 \quad \text { as } \quad \epsilon \rightarrow 0 .
$$

From (14), (15) and the explicit formulae (8), (11) for the construction of complex geometrical optics solutions, we have

$$
\int_{\Omega} q\left(a^{2}+\bar{a}^{2}\right) d x=0
$$

Computing the remaining terms, we have:

$$
\begin{array}{r}
K(\epsilon, \tau)+\frac{1}{\tau} \int_{\Omega} q\left(a\left(a_{0, \epsilon}+b_{0, \epsilon}\right)+\overline{a\left(a_{1, \epsilon}+b_{1, \epsilon}\right)}\right) d x+\int_{\Omega} q\left(a \bar{a} e^{2 \tau i \psi}+a \bar{a} e^{-2 \tau i \psi}\right) d x \\
+\frac{1}{4 \tau} \int_{\Omega}\left(q a \frac{\partial_{\bar{z}}^{-1}\left(a q_{2, \epsilon}\right)-M_{2, \epsilon}}{\partial_{z} \Phi}+q \bar{a} \frac{\partial_{z}^{-1}\left(q_{2, \epsilon} \bar{a}\right)-M_{4, \epsilon}}{\overline{\partial_{z} \Phi}}\right) d x \\
-\frac{1}{4 \tau} \int_{\Omega}\left(q a \frac{\partial_{\bar{z}}^{-1}\left(q_{1, \epsilon} a\right)-M_{1, \epsilon}}{\partial_{z} \Phi}+q \bar{a} \frac{\partial_{z}^{-1}\left(q_{1, \epsilon} \bar{a}\right)-M_{3, \epsilon}}{\partial_{z} \Phi}\right) d x \\
+o\left(\frac{1}{\tau}\right)=0 \quad \text { as } \tau \rightarrow+\infty
\end{array}
$$

Since the functions $q_{j}$ are not supposed to be from $C^{2}(\bar{\Omega})$, we can not directly use the stationary phase argument (e.g., Evans [8]). Consider two cases. Assume that $q \in W_{p}^{1}(\Omega)$ with $p>2$. We have

$$
\int_{\Omega} q \operatorname{Re}\left(a \bar{a} e^{2 \tau i \psi}\right) d x=\int_{\Omega} q_{\epsilon} \operatorname{Re}\left(a \bar{a} e^{2 \tau i \psi}\right) d x+\int_{\Omega}\left(q-q_{\epsilon}\right) \operatorname{Re}\left(a \bar{a} e^{2 \tau i \psi}\right) d x .
$$

We set $q_{\epsilon}=q_{1, \epsilon}-q_{2, \epsilon}$. Taking into account that $q_{j, \epsilon}=q_{j}$ on $\mathcal{H}, j=1,2$, (4) and using the stationary phase argument, similar to [11, we compute

$$
\int_{\Omega} q_{\epsilon}\left(a \bar{a} e^{2 \tau i \psi}+a \bar{a} e^{-2 \tau i \psi}\right) d x=\frac{2 \pi\left(q|a|^{2}\right)(\widetilde{x}) \operatorname{Re} e^{2 \tau i \operatorname{Im} \Phi(\widetilde{x})}}{\tau\left|\left(\operatorname{det} \operatorname{Im} \Phi^{\prime \prime}\right)(\widetilde{x})\right|^{\frac{1}{2}}}+o\left(\frac{1}{\tau}\right) \quad \text { as } \tau \rightarrow+\infty .
$$

For the second integral in (17) we obtain

$$
\begin{gathered}
\int_{\Omega}\left(q-q_{\epsilon}\right)\left(a \bar{a} e^{2 \tau i \psi}+a \bar{a} e^{-2 \tau i \psi}\right) d x=\int_{\Omega}\left(q-q_{\epsilon}\right)\left(a \bar{a} \frac{(\nabla \psi, \nabla) e^{2 \tau i \psi}}{2 \tau i|\nabla \psi|^{2}}-a \bar{a} \frac{(\nabla \psi, \nabla) e^{-2 \tau i \psi}}{2 \tau i|\nabla \psi|^{2}}\right) d x \\
=\int_{\partial \Omega}\left(q-q_{\epsilon}\right)\left(a \bar{a} \frac{(\nabla \psi, \nu) e^{2 \tau i \psi}}{2 \tau i|\nabla \psi|^{2}}-a \bar{a} \frac{(\nabla \psi, \nu) e^{-2 \tau i \psi}}{2 \tau i|\nabla \psi|^{2}}\right) d \sigma \\
-\frac{1}{2 \tau i} \int_{\Omega}\left\{e^{2 \tau i \psi} \operatorname{div}\left(\left(q-q_{\epsilon}\right) a \bar{a} \frac{\nabla \psi}{|\nabla \psi|^{2}}\right)-e^{-2 \tau i \psi} \operatorname{div}\left(\left(q-q_{\epsilon}\right) a \bar{a} \frac{\nabla \psi}{|\nabla \psi|^{2}}\right)\right\} d x
\end{gathered}
$$

Since $\left.\psi\right|_{\Gamma_{0}}=0$ we have

$$
\int_{\partial \Omega}\left(q-q_{\epsilon}\right) a \bar{a}\left(\frac{(\nabla \psi, \nu) e^{2 \tau i \psi}}{2 \tau i|\nabla \psi|^{2}}-\frac{(\nabla \psi, \nu) e^{-2 \tau i \psi}}{2 \tau i|\nabla \psi|^{2}}\right) d \sigma=\int_{\widetilde{\Gamma}} \frac{\left(q-q_{\epsilon}\right) a \bar{a}}{2 \tau i|\nabla \psi|^{2}}(\nabla \psi, \nu)\left(e^{2 \tau i \psi}-e^{-2 \tau i \psi}\right) d \sigma .
$$


By (44) and Proposition 2.4 in [1] we have that

$$
\int_{\partial \Omega}\left(q-q_{\epsilon}\right) a \bar{a}\left(\frac{(\nabla \psi, \nu) e^{2 \tau i \psi}}{2 \tau i|\nabla \psi|^{2}}-\frac{(\nabla \psi, \nu) e^{-2 \tau i \psi}}{2 \tau i|\nabla \psi|^{2}}\right) d \sigma=o\left(\frac{1}{\tau}\right) \quad \text { as } \tau \rightarrow+\infty .
$$

The last integral over $\Omega$ in formula (19) is $o\left(\frac{1}{\tau}\right)$ and so

$$
\int_{\Omega}\left(q-q_{\epsilon}\right)\left(a \bar{a} e^{2 \tau i \psi}+a \bar{a} e^{-2 \tau i \psi}\right) d x=o\left(\frac{1}{\tau}\right) \quad \text { as } \tau \rightarrow+\infty .
$$

Taking into account that $\psi(\widetilde{x}) \neq 0$ and using (26), (20) we have from (16) that

$$
\frac{2 \pi\left(q|a|^{2}\right)(\widetilde{x})}{\left|\left(\operatorname{det} \operatorname{Im} \Phi^{\prime \prime}\right)(\widetilde{x})\right|^{\frac{1}{2}}}+\widetilde{C}(\epsilon)=0
$$

where $\widetilde{C}(\epsilon) \rightarrow+0$ as $\epsilon \rightarrow 0$. Hence

$$
q(\widetilde{x})=0 \quad \text { if } a(\widetilde{x}) \neq 0 \text { and } a(x)=0 \text { for } x \in \mathcal{H} \backslash\{\widetilde{x}\} .
$$

Since a point $\tilde{x}$ can be chosen arbitrarily close to any given point in $\Omega$ (see [11]), we have $q \equiv 0$, that is, the proof of the theorem is completed if $q_{1}, q_{2} \in W_{p}^{1}(\Omega)$.

Fourth Step.

Now let $q \in C^{\alpha}(\bar{\Omega})$ with some $\alpha \in(0,1)$ and $\partial \Omega=\widetilde{\Gamma}$.

We recall the following classical result of Hörmander [9]. Consider the "oscillatory integral operator"

$$
T_{\tau} f(x)=\int_{\Omega} e^{-\tau i \psi(x, y)} a(x, y) f(y) d y,
$$

where $\psi \in C^{\infty}\left(\mathbb{R}^{2} \times \mathbb{R}^{2}\right)$ and $a(\cdot, \cdot) \in C_{0}^{\infty}\left(\mathbb{R}^{2} \times \mathbb{R}^{2}\right)$. We introduce the following matrix

$$
H_{\psi}=\left\{\partial_{x_{i} y_{j}}^{2} \psi\right\}
$$

Theorem 2 Suppose that $\operatorname{det} H_{\psi} \neq 0$ on suppa. Then

$$
\left\|T_{\tau}\right\|_{L^{2} \rightarrow L^{2}} \leq \frac{C}{\tau}
$$

Consider our holomorphic function $\Phi(x, y)=\left(x_{1}+i x_{2}-\left(y_{1}+i y_{2}\right)\right)^{2}+i$. We set $\psi(x, y)=2\left(x_{1}-y_{1}\right)\left(x_{2}-\right.$ $\left.y_{2}\right)-1$. Then

$$
H_{\psi}(x, y)=\left(\begin{array}{cc}
0 & -2 \\
-2 & 0
\end{array}\right)
$$

and $\operatorname{det} H_{\psi}(x, y)=-4$. Then the condition in Theorem 2 holds true.

We set $a(x, y)=\chi(x) \chi(y)$ where $\chi \in C_{0}^{\infty}\left(\mathbb{R}^{n}\right)$ and $\left.\chi\right|_{\Omega} \equiv 1$. Then, by Theorem 2 there exists a constant $C$ independent of $\tau$ such that

$$
\left\|T_{\tau}\right\|_{L^{2} \rightarrow L^{2}}+\left\|T_{-\tau}\right\|_{L^{2} \rightarrow L^{2}} \leq C / \tau \text {. }
$$

Setting $f=\left(q-q_{\epsilon}\right) a \bar{a} \chi_{\Omega}$ by (23) we have

$$
\left\|T_{\tau} f\right\|_{L^{2}(\Omega)}+\left\|T_{-\tau} f\right\|_{L^{2}(\Omega)} \leq C(\epsilon) / \tau, \quad C(\epsilon) \rightarrow 0 \quad \text { as } \quad \epsilon \rightarrow+0 .
$$

Therefore, by (24), in the ball $B(\widetilde{x}, \delta) \equiv\{x ;|x-\widetilde{x}|<\delta\}$, there exists a sequence of points $y(\tau)$ such that

$$
\left|\left(T_{\tau}\right) f(y(\tau))\right|+\left|\left(T_{-\tau}\right) f(y(\tau))\right| \leq \frac{C \epsilon}{\tau \delta^{2}} .
$$


Let $y(\tau)=\left(y_{1}(\tau), y_{2}(\tau)\right) \rightarrow \hat{y}(\epsilon)$ as $\tau \rightarrow+\infty$. By the stationary phase argument taking into account that $\psi(\widetilde{x}, \widetilde{x})=-1$, we have

$$
\int_{\Omega}\left(q_{\epsilon}-\left(q_{\epsilon}-q\right)(y(\tau)) \operatorname{Re}\left\{a \bar{a} e^{-2 \tau i \psi(y(\tau), x)}\right\} d x=\frac{2 \pi\left(q|a|^{2}\right)(\hat{y}(\epsilon)) \operatorname{Re} e^{2 \tau i}}{\tau}+o\left(\frac{1}{\tau}\right) .\right.
$$

From (16), (26), (25) we obtain

$$
2 \pi\left(q|a|^{2}\right)(\hat{y}(\epsilon)) \operatorname{Re} e^{2 \tau i}+\widetilde{C}(\epsilon)=0,
$$

where $\varlimsup_{\tau \rightarrow+\infty}|\widetilde{C}(\epsilon)| \rightarrow+0$ as $\epsilon \rightarrow 0$. Therefore as $\epsilon$ goes to zero, we have

$$
q(\hat{x})=0 .
$$

Here $\hat{x} \in B(\widetilde{x}, \delta)$ such that $\hat{y}(\epsilon) \rightarrow \hat{x}$ as $\epsilon \rightarrow+0$. Since $\delta>0$ and $\widetilde{x}$ are chosen arbitrarily, we conclude that $q \equiv 0$ in $\Omega$. Thus the proof of the theorem is completed.

\section{References}

[1] K. Astala and L. Päivärinta, Calderón's inverse conductivity problem in the plane, Ann. of Math., 163 (2006), 265-299.

[2] E. Blasten, The inverse problem of the Shrödinger equation in the plane. A dissection of Bukhgeim's result. ArXiv 1103.6200

[3] R. Brown and R. Torres, Uniqueness in the inverse conductivity problem for conductivities with $3 / 2$ derivatives in $L^{p}, p>2 n$, J. Fourier Analysis Appl. 9 (2003), 1049-1056.

[4] R. Brown and G. Uhlmann, Uniqueness in the inverse conductivity problem with less regular conductivities in two dimensions, Comm. Partial Differential Equations, 22 (1997), 1009-1027.

[5] A. Bukhgeim, Recovering the potential from Cauchy data in two dimensions, J. Inverse Ill-Posed Probl., 16 (2008), 19-34.

[6] A. Bukhgeim and G. Uhlmann, Recovering a potential from partial Cauchy data, Comm. Partial Differential Equations, 27 (2002), 653-668.

[7] A. P. Calderón, On an inverse boundary value problem, in Seminar on Numerical Analysis and its Applications to Continuum Physics, 65-73, Soc. Brasil. Mat., Río de Janeiro, 1980.

[8] L. Evans, Partial Differential Equations, American Mathematical Society, Providence, RI, 2000.

[9] L. Hörmander, The Analysis of Linear Partial Differential Operators I, Springer-Verlag, Berlin, 1985.

[10] C. Guillarmou and L. Tzou, Calderon inverse problem with partial Cauchy data on Riemannian surfaces, to appear in Duke Math. J., arXiv: 0908.1417v2.

[11] O. Imanuvilov, G. Uhlmann and M. Yamamoto, The Calderón problem with partial data in two dimensions, J. Amer. Math. Soc., 23 (2010), 655-691.

[12] O. Imanuvilov, G. Uhlmann and M. Yamamoto, Partial Cauchy data for general second order elliptic operators in two dimensions, arXiv:1010.5791 1

[13] C. Kenig, J. Sjöstrand and G. Uhlmann, The Calderón problem with partial data, Ann. of Math., 165 (2007), 567-591.

[14] A. Nachman, Global uniqueness for a two-dimensional inverse boundary value problem, Ann. of Math., 143 (1996), 71-96. 
[15] L. Päivärinta, A. Panchenko and G. Uhlmann, Complex geometrical optics for Lipschitz conductivities, Revista Matemática Iberoamericana, 19 (2003), 57-72.

[16] J. Sylvester and G. Uhlmann, A global uniqueness theorem for an inverse boundary value problem, Ann. of Math., 125 (1987), 153-169. 